\title{
Prediction of ground-borne vibration generated at railway crossings using a hybrid model
}

\author{
Evangelos Ntotsios ${ }^{1}$, Dimitrios Kostovasilis ${ }^{2}$, David Thompson ${ }^{1}$, Giacomo \\ Squicciarini ${ }^{1}$, and Yann Bezin ${ }^{2}$ \\ ${ }^{1}$ Institute of Sound and Vibration Research, University of Southampton \\ Southampton SO17 1BJ, United Kingdom \\ ${ }^{2}$ Institute of Railway Research, University of Huddersfield \\ Huddersfield HD1 3DH, United Kingdom
}

\begin{abstract}
Railway crossings are an important source of localized ground vibration. Models are required for identifying ways to tackle unacceptable levels of vibration from existing as well as future railway lines. Yet, the use of a prediction model that directly allows for the non-Hertzian wheel-rail contact dynamics and also includes three dimensional calculations of the ground response would be computationally expensive. In order to reduce the computational cost without affecting the accuracy of the predictions, a hybrid approach is proposed for the prediction of ground-borne vibration due to impacts at railway crossings. The approach combines the simulation of the vertical wheel-rail contact force in the time domain and the prediction of ground vibration levels in the far field using a linear wavenumber-frequency-domain approach. The proposed hybrid approach is used to investigate the influence of different vehicle speeds, crossing designs and wheel profiles on predicted ground vibration levels in the free field.
\end{abstract}

Keywords: Ground-borne Vibration, Railway Crossings, Hybrid Model, Equivalent Rail Unevenness.

\section{Introduction}

Turnouts (switches and crossings) are key elements of the railway system. A wheel passing over a railway crossing results in a high frequency impact load on the crossing nose (in the facing direction) or on the wing rail (in the trailing direction). The impact can cause wheel-rail contact fatigue and severe material degradation of the track components, but can also generate high levels of airborne noise and ground-borne vibration. This study addresses ground-borne vibration generated at a railway crossing that can cause annoyance (as either feelable vibration or re-radiated noise) or malfunctioning of sensitive equipment in nearby buildings.

In order to reduce the excessive levels of vibration that may be generated at railway crossings several empirical mitigation strategies have been proposed based on improved crossing designs [1], or installing vibration abatement measures on the track [2, 3]. Although the prediction of ground-borne vibration due to railway crossings is important for identifying ways to tackle unacceptable levels of vibration from existing as 
well as future railway lines, most studies that address wheel-rail interaction for complex rail profiles such as the crossing rail geometry are only focused on the prediction of wear and rolling-contact fatigue, rather than ground vibration. One reason is that the use of a prediction model that directly allows for the non-Hertzian wheel-rail contact and vehicle dynamics and also includes three dimensional calculations of the ground response would be highly computationally expensive [4]. In order to overcome the computational cost of such a complex model there have been a few studies in the literature that use a hybrid approach to predict ground-borne vibration due to impacts $[5,6$, 7], but these focus mainly on singular defects such as joints and wheel flats.

A hybrid method has been proposed for the prediction of impact noise due to excitation by wheel flats [8], and this was recently extended for excitation due to wheel-rail contact at crossings [9]. Based on this, a similar approach is proposed here to address the ground-borne vibration from crossings. In the proposed approach the simulations are performed in two steps: (i) calculation of impact loads by a time-domain simulation of non-linear dynamic vehicle-track interaction, and (ii) prediction of ground-borne vibration in the far field using the MOTIV model [10] in the frequency domain based on excitation by an "equivalent roughness" spectrum. The proposed model is used to investigate the influence of different vehicle speeds, crossing designs and wheel profiles on predicted ground vibration levels in the free field.

\section{Time-domain vehicle-crossing interaction model}

Simulation of the vibration due to the impacts requires accurate prediction of the magnitude and frequency content of the wheel-rail impact force. In the current work, dynamic vehicle-crossing interaction in the frequency interval up to $250 \mathrm{~Hz}$ is considered in the time domain using the VI-Rail software [11].

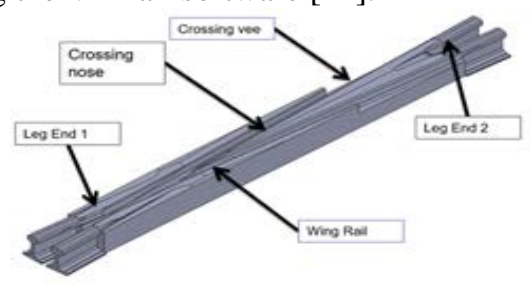

Fig. 1. The crossing geometry applied to the VI-Rail model.

In VI-Rail the track is considered as multiple rigid masses supported by vertical, lateral and rotational springs. VI-Rail models the track as a series of 'flexible moving' subsystems, that represent the left/right rails and sleeper/ballast as sprung masses. Every axle of the vehicle is connected to such a subsystem, having homogeneous properties and being independent from one another. For the vehicle, the software can take into account a detailed model including flexible modes and non-linearities; however, for the purpose of this study the vehicle model is considered as linear. The model includes the detailed rail profile at the crossing (Fig. 1) and calculates the wheel-rail contact forces along the rail due to the passage of the wheels. 


\section{Frequency-wavenumber domain model for ground vibration}

The MOTIV (ㅆodelling $\underline{\text { Of }}$ Train Induced Vibration) model [10] is used for the calculations of the vibration levels of the ground. This is a semi-analytical linear model for calculating ground vibration from surface and underground railways and for assessing the performance of vibration countermeasures at the track and/or the train. For surface railways, that are studied here, the model uses the wavenumber-frequency domain formulation originally developed by Sheng et al. [12] and extended in [13] to take into account the traction variation across the track-ground interface and to allow the excitation and response of the two rails to be considered separately.

A longitudinally invariant ballasted track is coupled vertically to the surface of the ground. The track structure is modelled as multiple beams supported by vertical springs with consistent mass and the soil is modelled as a horizontally layered halfspace as can be seen in Fig 2(a). Linear dynamic behaviour is assumed throughout. It is assumed that the wheels are always in contact with the rail and a linearized Hertzian contact spring is included between each wheel and the rail.

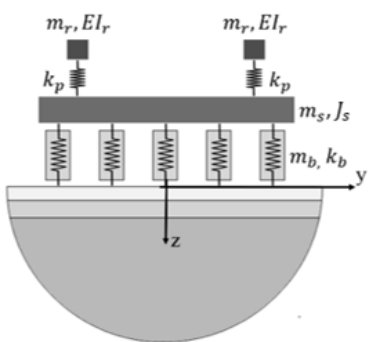

(a)

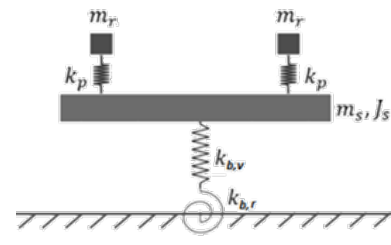

(b)

Fig. 2. Lateral view of the track multibody models for: (a) MOTIV; (b) equivalent VI-RAIL linear model.

For the ground vibration calculations only the vertical interaction forces are considered and only the vertical dynamics of the train are included. The vehicle is modelled as a linear 14-degree-of-freedom rigid-body system where the flexible modes of the car body are neglected. This is generally acceptable for ground vibration because the vehicle suspension isolates the car body above about $10 \mathrm{~Hz}$. To enable analysis in the frequency domain each non-linear suspension is linearized, which is valid for small motion amplitudes. In general, the model assumes that the excitation is due to the passage of individual wheel loads along the track (quasi-static loading) and due to dynamic interaction forces caused by irregularities of the wheels and tracks (dynamic loading).

\section{$4 \quad$ Hybrid method for predicting ground vibration at crossings}

Based on the equivalent roughness definition proposed in [8], a hybrid approach is developed to estimate the vibration levels of the ground from the contact forces calculated in the time domain. The idea is to find an equivalent roughness spectrum that, in a linear 
model, gives the same contact force spectrum as would be obtained with a non-linear model that includes the detailed rail profile and the discontinuity at the crossing. For this linear model, the receptances of the wheel and track must be the same as in the non-linear time-domain model but the non-linear properties of the contact are replaced by a linearised Hertzian contact spring. This roughness spectrum can then be used in a detailed linear vehicle-track-ground model, such as MOTIV [10], to predict the groundborne vibration.

\subsection{Equivalent roughness}

Within this framework, the first step is to calculate the transfer functions between roughness and contact forces given the vehicle, track and contact linear properties. By using a "moving roughness" approach, the contact force vector (at the two rails) $\mathbf{F}_{\mathbf{0}}(\omega)$ for a unit roughness on one of the two rails $\mathbf{u}_{0}=\left[\begin{array}{ll}1 & 0\end{array}\right]^{\mathrm{T}}$ can be obtained as

$$
\mathbf{F}_{\mathbf{0}}(\omega)=\left[\mathbf{R}_{\mathrm{R}}(\omega)+\mathbf{R}_{\mathrm{W}}(\omega)+\mathbf{R}_{\mathrm{C}}(\omega)\right]^{-1} \mathbf{u}_{0}
$$

where $\mathbf{R}_{\mathbf{i}}(\omega)$ is a $2 \times 2$ matrix of receptances of the rail ( $\mathrm{i}=\mathrm{R}$ ), wheel ( $\mathrm{i}=\mathrm{W}$ ) and contact $(\mathrm{i}=\mathrm{C})$

$$
\mathbf{R}_{\mathrm{i}}(\omega)=\left[\begin{array}{ll}
R_{\mathrm{i}, 11}(\omega) & R_{\mathrm{i}, 12}(\omega) \\
R_{\mathrm{i}, 21}(\omega) & R_{\mathrm{i}, 22}(\omega)
\end{array}\right]
$$

The rail and wheel receptances $R_{\mathrm{i}, k k}$ are the point receptances and $R_{\mathrm{i}, k l}$ with $(k=1,2$, $l=1,2$ and $k \neq l$ ) are transfer receptances from the contact point $k$ on one rail to the contact point $l$ on the other rail. These are calculated based on the properties of the time domain model presented in Sect. 2 by using a simplified linear track model to the VIRail track model that takes into account only the vertical dynamics of the track, as shown in Fig. 2(b), and vehicle. The contact receptance matrix $\mathbf{R}_{\mathrm{C}}(\omega)$ is a $2 \times 2$ diagonal matrix calculated from the axleload and the wheel radius that is constant for all frequencies $\omega$. The inverse of the matrix in Eq. (1) can be considered as the dynamic stiffness matrix of the vehicle-track linear system at the contact points.

Next, the time histories of the vertical contact forces for the two wheel-rail contact points of a single wheelset are calculated using the non-linear VI-Rail model described in Sect. 2. These are transformed into the frequency domain and collected in the vector $\mathbf{F}_{N}(\omega)$ using the fast Fourier transform. The equivalent roughness component on the crossing rail $u_{1}(\omega)$ is obtained as

$$
u_{1}(\omega)=\frac{F_{N, 1}(\omega)}{F_{0,1}(\omega)} .
$$

where $F_{0,1}(\omega)$ is from Eq. (1). For the second rail, the rail profile is assumed smooth and the equivalent roughness component $u_{2}(\omega)=0$.

\subsection{Sensitivity of equivalent roughness method to the track stiffness}

The rail point receptance $R_{\mathrm{R}, 11}(\omega)=R_{\mathrm{R}, 22}(\omega)$ of the linear track model is shown in Fig. 3(a). This is calculated using the linear track model of Fig. 1(b) using the same 
properties as the VI-Rail model in Table 1. Three different cases of track stiffness are shown in Fig. 3(a); for the reference case the ballast stiffness has the value reported in Table 1, but for the other two cases it has half or double that value. The wheel point receptance $R_{\mathrm{W}, 11}(\omega)=R_{\mathrm{W}, 22}(\omega)$ is also shown. This is calculated using the linear model from [13] based on the vehicle properties reported in [14]. For validation purposes the rail receptance calculated with the MOTIV model (Fig 2(a)) is also shown in Fig. 3(a). This uses the MOTIV track properties given in Table 1 that are given per unit length. It includes a ground with a $1 \mathrm{~m}$ deep soft soil surface layer with a shear wave (S-wave) speed of $190 \mathrm{~m} / \mathrm{s}$ and dilatational wave (P-wave) speed $420 \mathrm{~m} / \mathrm{s}$ and a stiffer half-space substratum with shear wave speed of $660 \mathrm{~m} / \mathrm{s}$ and dilatational wave speed of $1350 \mathrm{~m} / \mathrm{s}$. Damping is included in both materials as a loss factor of 0.1 . The receptance is similar to the one from VI-Rail up to $50 \mathrm{~Hz}$.

Table 1. Track properties.

\begin{tabular}{|c|c|c|c|}
\hline & & VI-Rail & MOTIV \\
\hline \multirow{2}{*}{ Rail } & Bending stiffness per rail & - & $1.5 \mathrm{MN} \mathrm{m}^{2}$ \\
\hline & Mass per unit length per rail & $195 \mathrm{~kg}$ & $195 \mathrm{~kg} / \mathrm{m}$ \\
\hline \multirow{4}{*}{ Rail pad } & Vertical stiffness per rail & $530 \mathrm{MN} / \mathrm{m}$ & $530 \mathrm{MN} / \mathrm{m}^{2}$ \\
\hline & Vertical damping coefficient & $350 \mathrm{kN} \mathrm{s} / \mathrm{m}$ & $350 \mathrm{kN} \mathrm{s} / \mathrm{m}^{2}$ \\
\hline & Lateral stiffness per rail & $430 \mathrm{MN} / \mathrm{m}$ & - \\
\hline & Lateral damping coefficient & $240 \mathrm{kN} \mathrm{s} / \mathrm{m}$ & - \\
\hline \multirow{2}{*}{ Sleeper } & Mass & $1200 \mathrm{~kg}$ & $1200 \mathrm{~kg} / \mathrm{m}$ \\
\hline & Pitching moment of inertia & $200 \mathrm{~kg} \mathrm{~m}^{2}$ & $200 \mathrm{~kg} \mathrm{~m}$ \\
\hline \multirow{8}{*}{ Ballast } & Mass & 0 & 0 \\
\hline & Vertical stiffness & $390 \mathrm{MN} / \mathrm{m}$ & $980 \mathrm{MN} / \mathrm{m}^{2}$ \\
\hline & Vertical damping coefficient & $480 \mathrm{kN} \mathrm{s} / \mathrm{m}$ & $480 \mathrm{kN} \mathrm{s} / \mathrm{m}^{2}$ \\
\hline & Lateral stiffness & $37 \mathrm{MN} / \mathrm{m}$ & - \\
\hline & Lateral damping coefficient & $480 \mathrm{kN} \mathrm{s} / \mathrm{m}$ & - \\
\hline & Rotational stiffness & $10 \mathrm{MN} \mathrm{m} / \mathrm{rad}$ & - \\
\hline & Rotational damping coefficient & $10 \mathrm{kN} \mathrm{m} \mathrm{s} / \mathrm{rad}$ & - \\
\hline & Ballast/ground interaction width & - & $3.2 \mathrm{~m}$ \\
\hline
\end{tabular}

The contact forces at the crossing rail that are calculated with the VI-Rail model (using the properties reported in [14] for the vehicle and Table 1 for the track) and the equivalent roughness spectra in one-third octave band wavelengths calculated using Eq. (3) are shown in Fig. 3(b) and Fig. 3(c) respectively. The results are obtained for a train speed of $22.2 \mathrm{~m} / \mathrm{s}(80 \mathrm{~km} / \mathrm{h})$ and for a contact spring stiffness of $1.11 \mathrm{GN} / \mathrm{m}$.

In Fig. 3(b) and Fig 3(c) results are shown for the three different values of ballast stiffness; in Fig 3(c) the case in which the MOTIV track model used for the calculation of the rail receptance is also shown. It can be seen that for the different cases of track stiffness, although the rail receptances in Fig. 3(a) are significantly different, the calculated contact forces are very similar and the estimated equivalent roughness spectra are almost identical. This suggests that the contact forces at the crossing contact points depend mostly on the contact conditions and not on the track stiffness. 

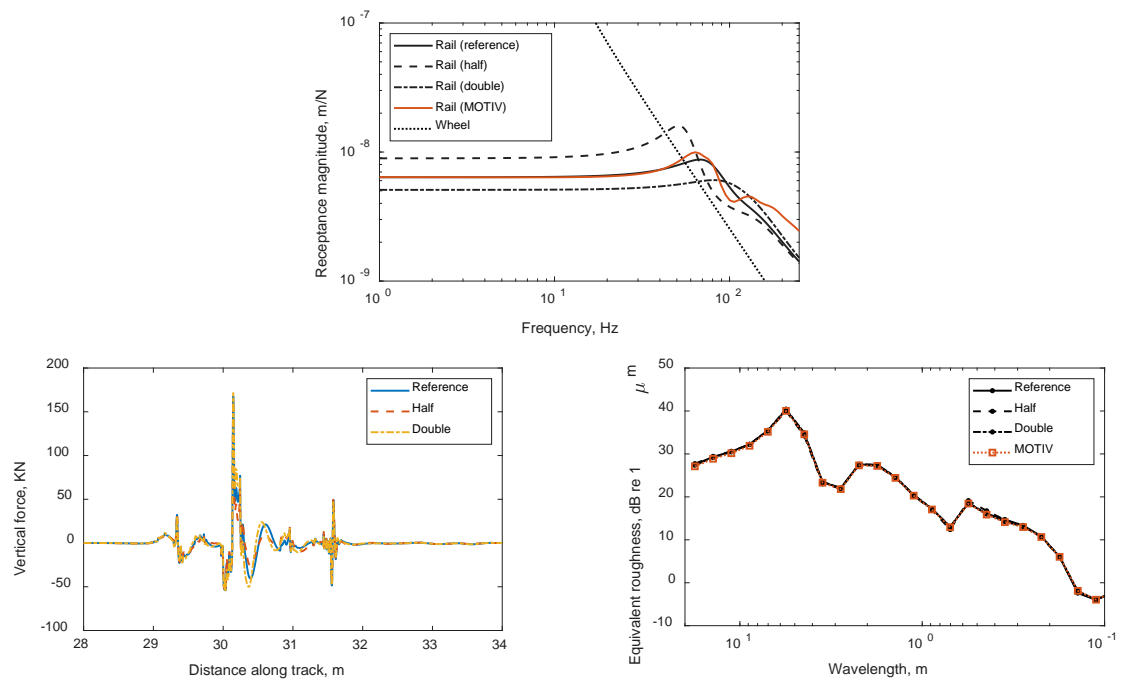

Fig. 3. (a) Rail and wheel receptance magnitudes; (b) contact forces; (c) equivalent roughness.

\section{$5 \quad$ Results}

The passage of the benchmark vehicle [14] is considered with speed $80 \mathrm{~km} / \mathrm{h}$ in the facing move, from the switch panel towards the crossing panel of the through route. The track properties for the VI-Rail model and the MOTIV model are given in Table 1. For the ground model the properties that are used are the same as those reported in Sect. 4.2. Any further track irregularity outside the crossing region is not considered here even if it is allowed for by the simulation procedure.

Fig. 4 compares the predicted ground vibration levels from the hybrid model for different speeds, worn wheel profiles and crossing designs. The results are given in terms of the one-third octave spectrum of the predicted vertical velocity level of the ground surface, calculated at $16 \mathrm{~m}$ from the track due to the passage of the train over the crossing panel.

The effect of the speed on the contact forces and the ground vibration levels shown in Figs. 4(a) and 4(b) is significant. An increase in vehicle speed from 80 to $160 \mathrm{~km} / \mathrm{h}$ corresponds to an increase in the peak contact force. The vibration levels at low frequencies also increase due to the effect of the quasi-static response [13]. In addition, a decrease in the maximum vibration levels is observed at frequencies around the P2 resonance (at about $60 \mathrm{~Hz}$ ). This is caused by the wheel having a smaller vertical drop at the crossing at the higher speed due to its inertia. Similar findings are reported in [6].

The influence of the worn wheel profile on the impact force magnitude and associated ground vibration level shown in Figs. 4(c) and 4(d) is also significant. For the selected worn wheel profile, it is shown that wheel wear can increase the contact forces and also the vibration levels in the free field for a wide range of frequencies.

The different crossing type considered in Figs. 4(e) and 4(f) also affects the contact forces and ground vibration level. However, although it seems to have increased the 
contact forces, the predicted vibration levels are lower between 16 and $125 \mathrm{~Hz}$, where the dynamic contribution in the total response is significant.

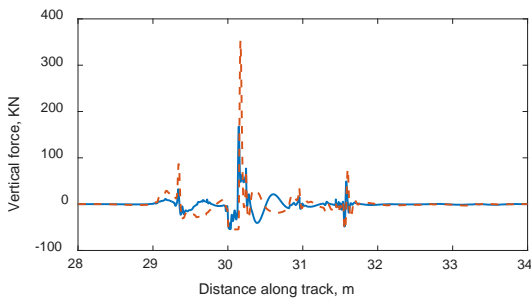

(a)

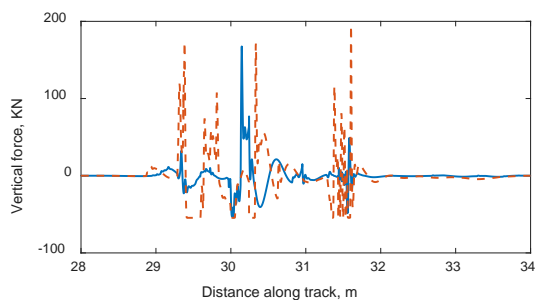

(c)

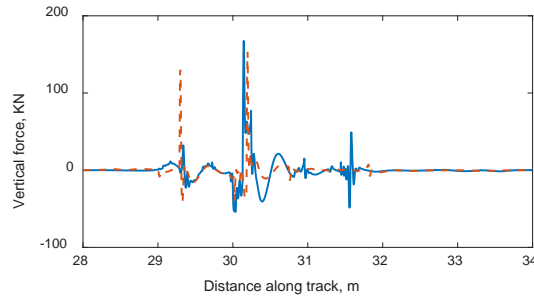

(e)

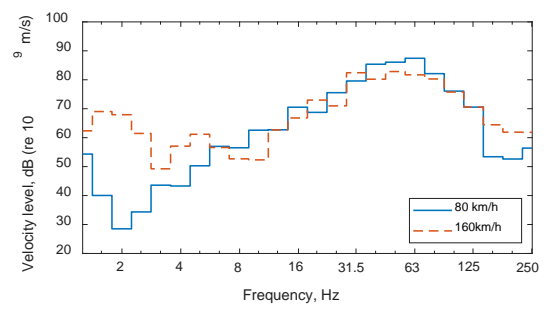

(b)

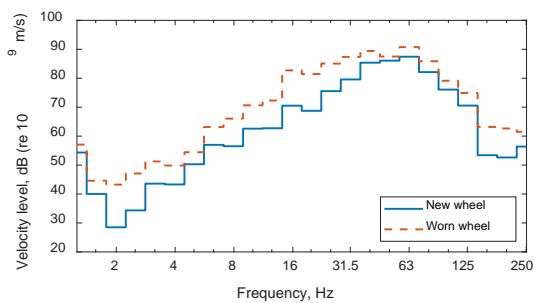

(d)

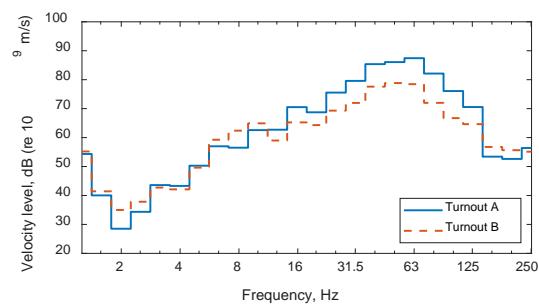

(f)

Fig. 4. Comparison of (a, c, e) contact forces and (b, d, f) ground vibration levels at $16 \mathrm{~m}$ from the track for different (a, b) train speeds; (c, d) wheel wear profiles; (e, f) crossing types.

\section{Conclusions}

A model for the prediction of ground-borne vibration at railway crossings has been developed. The hybrid approach based on the concept of an equivalent roughness spectrum is used in order to combine two already existing and validated prediction models: VI-Rail for numerical simulation of non-linear time-domain dynamic vehicle-track interaction, and the linear wavenumber-frequency domain model MOTIV for the prediction of ground vibration.

Based on the simulations it is concluded that the vibration levels are affected by train speed, wheel wear or crossing design in the higher frequencies of the spectrum, where the dynamic contribution in the total response is significant. At lower frequencies, the total vibration is influenced by the train speed due to its effect on the quasi-static component of the response. It is also affected by the selected worn wheel profile, which 
increases the low frequency components of the contact forces. These findings suggest that, when comparing predictions with measurements, uncertainties in wheel and rail profiles can influence the outcome considerably.

\section{Acknowledgements}

The financial support by the EPSRC under the grant EP/M025276/1 is gratefully acknowledged.

\section{References}

1. Kaewunruen, S.: Effectiveness of Using Elastomeric Pads to Mitigate Impact Vibration at an Urban Turnout Crossing. In: Maeda T. et al. (eds) Noise and Vibration Mitigation for Rail Transportation Systems. Notes on Numerical Fluid Mechanics and Multidisciplinary Design, vol. 118, pp. 357-365. Springer, Tokyo (2012).

2. Talbot, J.P.: Lift-over crossings as a solution to tram-generated ground-borne vibration and re-radiated noise. Proceedings of the Institution of Mechanical Engineers, Part F: Journal of Rail and Rapid Transit 228(8), 878-886 (2014).

3. Müller, R., Nielsen, J.C.O., Nélain, B., Zemp, A.: Ground-Borne Vibration Mitigation Measures for Turnouts: State-of-the-Art and Field Tests. In: Nielsen J. et al. (eds) Noise and Vibration Mitigation for Rail Transportation Systems. Notes on Numerical Fluid Mechanics and Multidisciplinary Design, vol. 126, pp. 547-554. Springer, Berlin, Heidelberg (2015).

4. Nielsen J., Pieringer A., Thompson D., Torstensson P.: Wheel-rail impact loads, noise and vibration: A review of excitation mechanisms, predidction methods and mitigation measures. Noise and Vibration Mitigation for Rail Transportation Systems - Proceedings of the 13th International Workshop on Railway Noise, 16-20 September 2019, Ghent, Belgium.

5. Nielsen, J.C.O., Lombaert, G., Francois, S.: A hybrid model for prediction of ground-borne vibration due to discrete wheel/rail irregularities. Journal of Sound and Vibration 345, 103120 (2015).

6. Kouroussis, G., Connolly, D.P., Alexandrou, G., Vogiatzis, K.: Railway ground vibrations induced by wheel and rail singular defects. Veh Sys Dynamics 53(10), 1500-1519 (2015).

7. Connolly, D.P., Galvín, P., Olivier, B., Romero, A., Kouroussis, G.: A 2.5D time-frequency domain model for railway induced soil-building vibration due to railway defects. Soil Dynamics and Earthquake Engineering 120, 332-44 (2019).

8. Wu, T.X., Thompson, D.J.: A hybrid model for the noise generation due to railway wheel flats, Journal of Sound and Vibration 251(1), 115-139 (2002).

9. Torstensson, P.T., Squicciarini, G., Krüger, M., Pålsson, B. A., Nielsen, J.C.O., Thompson, D. J.: Wheel-rail impact loads and noise generated at railway crossings - influence of vehicle speed and crossing dip angle. Journal of Sound and Vibration 456, 119-136 (2019).

10. MOTIV Software, https://motivproject.co.uk/motiv-software, last accessed 2019/05/30.

11. VI-RAIL, https://www.vi-grade.com/en/products/VI-Rail, last accessed 2019/05/30.

12. Sheng, X., Jones, C.J.C., Thompson, D.J.: A theoretical model for ground vibration from trains generated by vertical track irregularities. Journal of Sound and Vibration 272(3-5), 937-965 (2004).

13. Ntotsios, E., Thompson, D., Hussein, M.: The effect of track load correlation on groundborne vibration from railways. Journal of Sound and Vibration 402, 142-163 (2017).

14. Iwnicki, S.: Manchester benchmarks for rail vehicle simulation. Vehicle System Dynamics 30(3-4), 295-313 (1998). 